
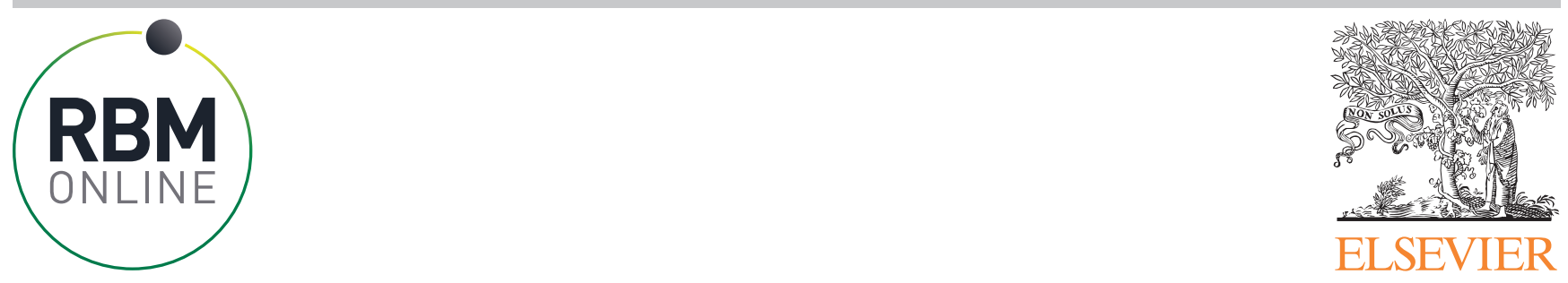

\title{
Article
}

\section{Effect of the antioxidant idebenone on maternal diabetes-induced embryo alterations during early organogenesis}

\author{
Romina Higa *, Sabrina Roberti, María Belén Mazzucco, Verónica White, \\ Alicia Jawerbaum \\ Laboratory of Reproduction and Metabolism, CEFYBO-CONICET, School of Medicine, University of Buenos Aires, \\ Paraguay 2155 (1121ABG), Buenos Aires, Argentina
}

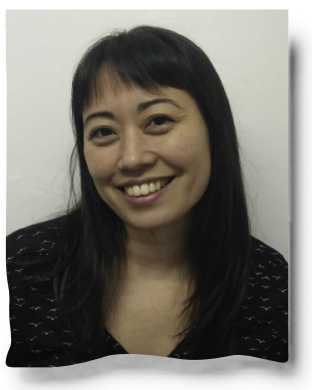

Romina Higa obtained her PhD from Buenos Aires University (UBA). She is Assistant Investigator at the Argentine National Research Council (CONICET) at the Laboratory of Reproduction and Metabolism (CEFyBO-CONICETUBA). Her research focus is the effect of maternal diabetes on embryo development and on putative treatments to prevent the diabetic embryopathy.

\section{KEY MESSAGE}

Rats with mild diabetes were used to investigate the usefulness of maternal treatments with idebenone to prevent post-implantation embryo impairments. A mitochondrial antioxidant maternal treatment prevented maternal diabetes-induced embryo alterations in prooxidant, proinflammatory and apoptotic markers, and in the expression of genes involved in mitochondrial biogenesis and function.

\section{A B S T R A C T}

Research question: Can maternal treatments with idebenone, a structural analogue of coenzyme Q10, prevent alterations on markers of proinflammatoryprooxidant processes, on the expression of genes involved in mitochondrial biogenesis and function, and on the apoptotic rate in embryos from mild diabetic rats?

Design: A mild diabetic rat model was induced by neonatal-streptozotocin administration $190 \mathrm{mg} / \mathrm{kg}$ subcutaneously). Female diabetic rats and controls were mated with healthy males. From day 1 of pregnancy, control and diabetic rats were orally treated with idebenone $(100 \mathrm{mg} / \mathrm{kg}$ daily). On day 10.5 of gestation, the embryos were explanted and prepared for immunohistochemical studies, for the evaluation of gene expression by reverse transcription polymerase chain reaction and for TdT (terminal deoxynucleotidyl transferase)-mediated dUDP nick-end-labelling assay analysis.

Results: Embryos from mild diabetic rats showed increased levels of nitrated proteins, 4-hydroxynonenal and matrix metalloproteinase 9, which were prevented by idebenone administration. We also found a decreased embryonic expression of cytochrome c oxidase and reduced mRNA levels of peroxisome proliferator activated receptor- $\gamma$ coactivator $-1-\alpha$ and nuclear respiratory factor- 1 , both of which were prevented by idebenone administration to the diabetic pregnant rats. Embryos from mild diabetic rats also showed an increased apoptotic rate, which was diminished by idebenone treatment. Conclusion: Maternal idebenone treatment ameliorates altered parameters related to the prooxidant-proinflammatory environment found in embryos from mild diabetic rats, suggesting a putative treatment to prevent diabetes-induced embryo alterations.

(c) 2018 Reproductive Healthcare Ltd. Published by Elsevier Ltd. All rights reserved.

* Corresponding author.

E-mail address: rominahigalagmail.com (R Higa).

https://doi.org/10.1016/j.rbmo.2018.05.006

1472-6483/@ 2018 Reproductive Healthcare Ltd. Published by Elsevier Ltd. All rights reserved. 


\section{Introduction}

In diabetic pregnancies, many molecular and cellular pathways are altered during embryo development. These alterations are related to hyperglycaemia and the increase in other intrauterine metabolic substrates, which lead to the generation of a proinflammatory and prooxidant environment (Eriksson and Wentzel, 2016). During early organogenesis, the developing embryo is especially susceptible to the diabetes-induced prooxidant environment caused by the immaturity of its antioxidant system (Zaken et al., 2000). Increased embryonic oxidative stress is involved in diabetic-induced teratogenicity, and further contributes to fetal alterations and the programming of diseases in offspring (Higa and Jawerbaum, 2013; Jawerbaum and White, 2017; Ornoy, 2007; Zabihi and Loeken, 2010; Zhao and Reece, 2013).

Reactive oxygen species mainly originate in the mitochondrion, an organelle whose function is altered in the development of adult diabetic complications (Blake and Trounce, 2014). Cytochrome c oxidase (COX), the terminal oxidase of the mitochondrial electron transport chain, catalyzes the final step of electron transfer from reduced cytochrome $c$ to oxygen to make water. It is also one of the three proton pumps that generate the proton gradient across the inner mitochondrial membrane, which powers adenosine triphosphate synthesis. The transcription of nuclear genes that encode COX subunits is regulated by nuclear respiratory factors 1 and 2 (NRF1 and NRF2) (Dhar et al., 2008). NRF1 and NRF2 also regulate most nuclear genes involved in mitochondrial biogenesis (mitochondrial transcription factors A, B1 and B2 [Tfam, Tfb $1 \mathrm{~m}$ and Tfb2m]). In this context, the role of peroxisome proliferator activated receptor- $\gamma$ coactivator $1-\alpha$ (PGC$1 \alpha$ ), which coactivates different transcription factors in response to energy requirements, is also important to promote the activation of the transcriptional machinery that regulates mitochondrial biogenesis. PGC- $1 \alpha$ coactivates NRF1 and NRF2 as well as peroxisome proliferator activated receptor $\delta$ (PPAR $\delta$ ) (Puigserver and Spiegelman, 2003; Scarpulla, 2008; Wang et al., 2003). This is a nuclear receptor that acts as a nutritional sensor and is able to bind a great variety of lipid molecules, triggering the transcription of genes involved in anti-inflammatory, anti-oxidant, metabolic and developmental processes (Coll et al., 2010; Rotman et al., 2006). It is the only PPAR isotype expressed in high levels in the embryo during early organogenesis (Braissant and Wahli, 1998). In embryos from pregestational streptozotocin-induced diabetic rats (with maternal glycaemia values over $350 \mathrm{mg} / \mathrm{dl}$, rat model of severe diabetes), PPAR $\delta$ expression is reduced and its ligands ameliorate several altered parameters in diabetic embryopathy (Higa et al., 2007, 2012).

Oxidative and nitrative stress have been shown in embryos from mild and severe experimental models of diabetes (Gareskog et al., 2006; Jawerbaum and Gonzalez, 2006; Sakamaki et al., 1999; Singh et al., 2011; Viana et al., 2003). Mitochondrial damage has been found in embryos from diabetic rats during early organogenesis, although this is a period characterized by active glycolytic pathways and immature mitochondrial metabolism (Akazawa, 2005; Yang et al., 1995). Antioxidants have been found to have positive effects on the prevention of experimental diabetic embryopathy (Sakamaki et al., 1999; Viana et al., 2003). The putative effect of antioxidants with mitochondrial action, such as idebenone (2-[10-Hydroxydecyl]-5,6-dimethoxy-3methyl-1,4-benzoquinone), a structural analogue of coenzyme Q10, and a potent antioxidant (Carbone et al., 2012; Geromel et al., 2002; Lin et al., 2015), has never been previously studied. Therefore, in this study, we used a rat model of mild diabetes Iglycaemia values below
$250 \mathrm{mg} / \mathrm{dl}$, induced by neonatal streptozotocin administration) to address the effect of idebenone on markers of inflammatoryoxidative processes, on the expression of genes involved in mitochondrial function biogenesis, and on apoptosis in the embryo during early organogenesis.

\section{Materials and methods}

\section{Animals}

Albino Wistar rats were bred in the laboratory with free access to commercial rat chow (Asociación Cooperativa Argentina, Buenos Aires, Argentinal and water, in a lighting cycle of $12 \mathrm{~h}$ light-12 h dark. A mild diabetic rat model was developed by injecting 2-day-old neonates with streptozotocin $190 \mathrm{mg} / \mathrm{kg}$, subcutaneously, Sigma-Aldrich, St Louis, MO, USA, diluted in citrate buffer $0.05 \mathrm{M} \mathrm{pH} \mathrm{4.5,} \mathrm{Sigma-Aldrich),} \mathrm{as}$ described previously (Jawerbaum and White, 2010; Martinez et al., 2012). Because streptozotocin has a short half-life at neutral $\mathrm{pH}$ and selectively enters and accumulates in beta-cells, its cytotoxicity is largely limited to these cells at the doses we used (Eleazu et al., 2013; Jawerbaum and White, 2010).

Control rats were injected with citrate buffer only. Glycaemia was measured with Accu-Chek reagent strips and a glucometer (Bayer Diagnostics, Buenos Aires, Argentinal and confirmed before mating. Pregnant rats were fasted for $6 \mathrm{~h}$ before glycaemia measurements were taken on day 10.5 of pregnancy. Rats were considered diabetic when they presented fasting glucose values higher than $130 \mathrm{mg} / \mathrm{dl}$. The reproductive characteristics of the diabetic model have been previously described (Jawerbaum and White, 2010). The animal protocol was approved on 24 July 2013 by the Institutional Committee for the Care and Use of Experimental Animals (CICUAL, Resolution CD N ${ }^{\circ}$ 1497/2013), School of Medicine, University of Buenos Aires, and conducted according to the Guide for the Care and Use of Laboratory Animals, US National Institutes of Health (NIH Publication, 8th Edition, 2011, http://www.ncbi.nlm.nih.gov/books/NBK54050/?report=reader).

\section{Animal treatment and tissue collection}

Control and diabetic female adult rats were mated with untreated adult males. Mating was confirmed by the presence of sperm in vaginal smears. The presence of sperm in vaginal smears was used to confirm mating and to designate day 0.5 of gestation. Animals received treatment with either idebenone (Sigma-Aldrich, $100 \mathrm{mg} / \mathrm{kg}$ ) or vehicle, orally from day $0.5-10.5$ of gestation.

Animals were killed in a $\mathrm{CO}_{2}$ chamber on day 10.5 of pregnancy, and the uteri were transferred to Petri dishes with Krebs Ringer Bicarbonate solution: $5 \mathrm{mM}$ glucose, $145 \mathrm{mM} \mathrm{Na}^{+}, 5.9 \mathrm{mM} \mathrm{K}^{+}, 2.2 \mathrm{mM}$ $\mathrm{Ca}^{+2}, 1.2 \mathrm{mM} \mathrm{Mg}^{+2}, 127 \mathrm{mM} \mathrm{Cl}^{-}, 25 \mathrm{mM} \mathrm{HCO}_{3}^{-}, 1.2 \mathrm{mM} \mathrm{SO}_{4}^{-2}$ and $1.2 \mathrm{mM}$ $\mathrm{PO}_{4}^{-3}$. The balls of decidual tissue were explanted from each uterus and gently opened to free the conceptuses, using a stereomicroscope and microsurgical dissecting instruments. The embryos were dissected out of the yolk sacs and evaluated morphologically under a stereomicroscope. On day 10.5 of pregnancy, the embryos of the evaluated rats are in the process of neural tube closure, as closure occurs on day 12 of pregnancy.

Viability was established by the presence of a beating heart. Malformed embryos (3\% in the diabetic group) received no further analysis. Non-malformed viable embryos were further processed according to protocols described below. 
Table 1 - Primer sequences and optimum cycle number for each primer pair used for gene expression analysis by semiquantitative polymerase chain reaction in rat embryo of 10.5 days of gestation.

\begin{tabular}{llll} 
Gene & $\begin{array}{l}\text { Forward primer } \\
\left.\text { (5' to } 3^{\prime}\right)\end{array}$ & $\begin{array}{l}\text { Reverse primer } \\
\left.\text { (5' to } 3^{\prime}\right)\end{array}$ \\
\hline CoxIVi1 & TACGAAACACGACCCCACTG & AGCTAGCGACTACGCTTTCC \\
Nrf1 & CAACAGGGAAGAACGGAA & GTGGCTCTGAGTTTCCGAAG & 28 \\
Tfam & CTGATGGCCATTACATGTGG & AAAGCCCGGAGGTTAG & 27 \\
Ppar $\delta$ & GAGGGGTCAAGGGCTTCTT & CACTTGTTGCGGTTCTTCTTCTG & 26 \\
Pgc- $1 \alpha$ & GTGCAGCCAAGACTCTGTATGG & GTCCAGGTCATTCACATCAAGTTC & 30 \\
L30 & CCATCTTGGCGTCTGATCTT & GGCGAGGATAACCAATTC & 34 \\
\hline
\end{tabular}

\section{Analysis of mRNA levels of cox, nrf1, tfam, ppar $\delta$ and pgc- $1 \alpha$}

The mRNA levels of Cox, Nrf1, Tfam, Ppar $\delta$ and Pgc-1 $\alpha$ were evaluated by semi-quantitative reverse transcription polymerase chain reaction (RT-PCR), as previously described (Higa et al., 2014). Four pooled embryos from each rat in each experimental group $(n=9)$ were kept in RNAlater ${ }^{\circledR}$ (Ambion, Texas, USA) at $-20^{\circ} \mathrm{C}$ and RNA was extracted with RNAzol (Molecular Research Center, Cincinnati, USA) in accordance with the manufacturer's instructions. cDNA was synthesized by incubating $2 \mu \mathrm{g}$ of extracted RNA in a first-strand buffer containing Moloney murine leukemia virus (MMLV) enzyme from Promega (Buenos Aires, Argentina), random primer hexamers (Promega) and each of all four deoxynucleotide triphosphates (Invitrogen, San Diego, CA, USA), in accordance with the MMLV manufacturer's instructions (final volume $25 \mu \mathrm{l}$ ). The reaction mixture was incubated at $37^{\circ} \mathrm{C}$ for $60 \mathrm{~min}$ and then at $70^{\circ} \mathrm{C}$ for $15 \mathrm{~min}$. cDNA $(2 \mu \mathrm{l}$, selected to work within the linear rangel was amplified by polymerase chain reaction in a buffer containing deoxynucleotide triphosphates, magnesium chloride solution, Taq polymerase (GoTaq Polymerase, Promega, Wisconsin, USA) and each specific primer, in accordance with the Taq polymerase manufacturer's instructions. The primers used (Table 1), were purchased from Invitrogen. The primers for the ribosomal protein L30 were used as an internal control.

The initial step in the reaction was $95^{\circ} \mathrm{C}$ for $5 \mathrm{~min}$. The following numbers of cycles are detailed in Table 1. Each cycle consisted of denaturation at $95^{\circ} \mathrm{C}$ for $15 \mathrm{~s}$ primer annealing at $58^{\circ} \mathrm{C}$ for $30 \mathrm{~s}$ and extension at $72^{\circ} \mathrm{C}$ for $15 \mathrm{~s}$. The resulting products were separated on a $2 \%$ agarose gel and stained with SYBR ${ }^{\circledR}$ Safe (Invitrogen). The images were taken with the ImageQuant image acquiring system and software (GE Healthcare, Buckinghamshire, UK) and quantified with the Image J software, National Institutes of Health, Bethesda, MD, USA.

\section{Immunohistochemical and TUNEL analysis}

Immunohistochemistry was carried out to determine levels and localize 4-hydroxynonenal, nitrotyrosine, matrix metalloproteinase 9 (MMP9) and subunit IV of COX (COXIV) in embryos, as described previously (Higa et al., 2011). Two embryos from each rat $(n=5)$ in each experimental group were explanted, immediately fixed in $4 \%$ phosphate buffered saline (PBS)-paraformaldehyde and then paraffinized. The same number of serial, $5-\mu \mathrm{m}$ sections were captured from each embryo and on each slide, providing reasonable confidence that protein localization and quantification were performed on comparable anatomical planes from each embryo. All sections were processed simultaneously under identical conditions. Sections were deparaffinized and hydrated in xylene and a series of graded ethanol solutions, followed by three washes in PBS and PBS with $0.05 \%$ Tween-20 (PBST). Incubation in $0.3 \%$ hydrogen peroxide in PBS to block endogenous peroxidase was carried out for $20 \mathrm{~min}$. Slides were incubated in a humidified chamber overnight with a rabbit anti-4-hydroxynonenal antibody (1:400 Abcam, Cambridge, UK), rabbit anti-nitrotyrosine antibody (1:4000, Millipore, Temecula, USA), a mouse anti-MMP9 antibody (1:150, Calbiochem, Billerica, USA) or a rabbit anti-COXIV antibody (1:300, Abcam). All primary antibodies were checked for antibody specificity and diluted in PBS-T with 1\% bovine serum albumin. Negative controls were carried out in the absence of primary antibody and by replacing the primary antibody with a pooled serum of the same species that contains a spectrum of the Immunoglobulin $G$ subclasses (Vector Laboratories Burlingame, CA, USA). Biotinylated horse anti-mouse antibody, rat adsorbed 1 1:200 in PBS-T/1.5\% normal horse serum) or biotinylated goat anti-rabbit 1:200 in PBS-T/1.5\% normal goat serum) (Vector Laboratories) was applied, followed by incubation with an avidin-biotin complex for $60 \mathrm{~min}$ (Vector Laboratories). Staining was visualized by adding $40 \% 3,3^{\prime}$-diaminobenzidine tetrahydrochloride chromogen-buffer plus $0.02 \%$ (v/v) hydrogen peroxide in $0.05 \mathrm{M}$ Tris (pH 7.6) for $10 \mathrm{~min}$. A positive staining appeared as a dark brown colour. The tissues were then counterstained with haematoxylin and dehydrated before mounting. Five sections of two embryos from each rat of each experimental group were examined by two skilled blinded observers under light microscopy (Nikon Eclipse E200) and photographed (Nikon DS-Fi1). Immunoreactivity intensity was quantified using the ImageProPlus software, Media Cybernetics, Bethesda, MD, USA. Data are shown as relative to a value of 1 assigned to the mean value for control. Similar results were obtained using a semiquantitative score (data not shown).

To study apoptosis in embryos, a TdT (terminal deoxynucleotidyl transferase)-mediated dUTP nick-end-labelling (TUNEL) assay was carried out using an in-situ cell apoptosis detection kit (DeadEnd Fluorometric TUNEL System, Promegal according to the manufacturer's instructions. The sections were examined under fluorescent microscopy (Nikon Eclipse E200) and photographed (Nikon DS-Fi1). The number of positive cells was quantified using the Image $J$ software. Data are shown as relative to a value of 1 assigned to the mean value for controls.

\section{Statistical analysis}

Data are presented as the mean \pm standard error. Groups were compared by two-way analysis of variance in conjunction with Bonferroni's test. In all cases, differences were considered statistically significant at $P<0.05$ 


\section{Results}

4-hydroxynonenal, nitrotyrosine and MMP9 levels in embryos from control and diabetic rats treated or not with idebenone

We first evaluated control and mild diabetic animals treated or not with idebenone during gestation and found that glycaemia was increased in the diabetic group (control: $101 \pm 7 \mathrm{mg} / \mathrm{dl}$; diabetic: 213 $\pm 29 \mathrm{mg} / \mathrm{dl}, P<0.001)$ compared with the control group and that idebenone treatment was unable to reduce the increased maternal glucose values (control treated with idebenone: $98 \pm 9 \mathrm{mg} / \mathrm{dl}$; diabetic treated with idebenone: $207 \pm 33 \mathrm{mg} / \mathrm{dll}$ ). Oxidative stress during early organogenesis can lead to adverse developmental outcomes and disease risk later in life. We, therefore, studied 4-hydroxynonenal levels as a stable product of oxidative-stress induced lipid peroxidation, and found that its levels were increased in embryos from mild diabetic rats compared with embryos from control rats $(P<0.001)$ (Figure 1),

a)

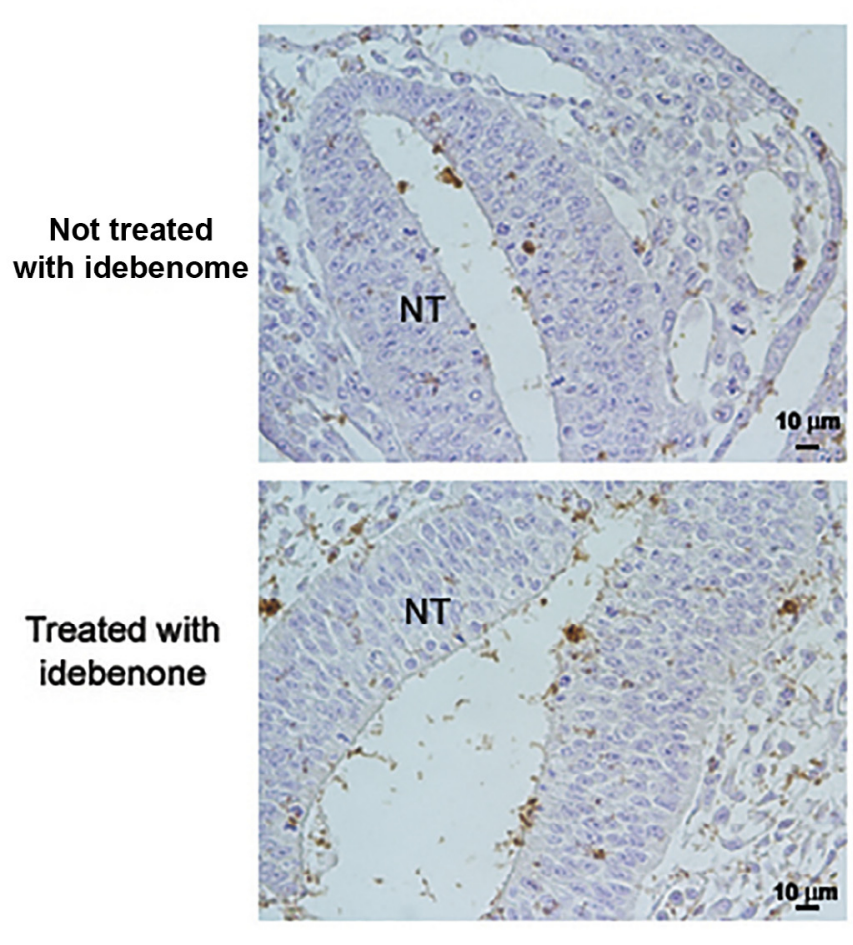

Control
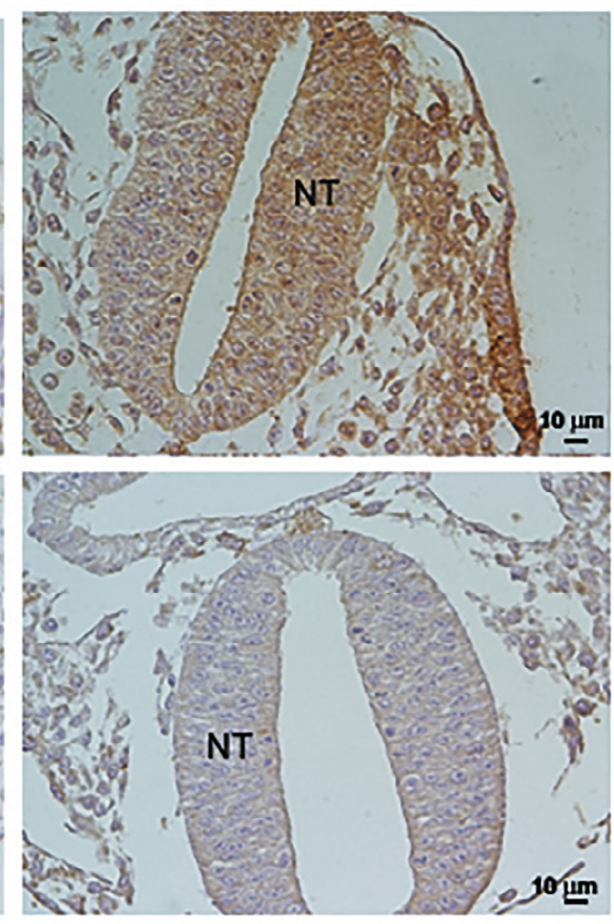

b)

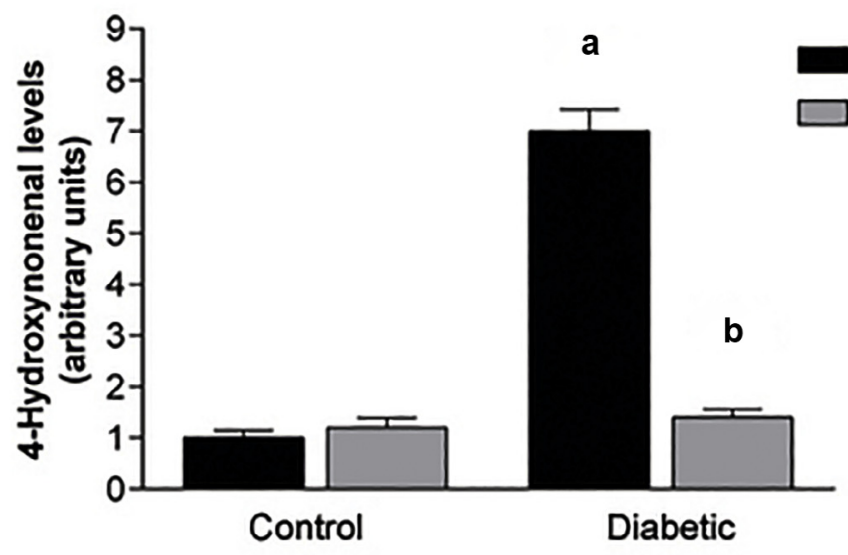

Figure 1 - Effect of maternal idebenone treatment on the levels of 4-hydroxynonenal in embryos from control and diabetic rats on day 10.5 of gestation. (a) A representative photomicrograph of a transverse section from the cephalic zone of the embryo (400X) stained with a specific anti-4-hydroxynonenal antibody; (b) graphs quantifying the immunohistochemical staining represented in (a). Values are means \pm SEM ( $n=5$ rats). Two-way analysis of variance in conjunction with Bonferroni's test was carried out. ${ }^{a} P<0.001$ versus control rats not treated with idebenone; ${ }^{\mathrm{b}} \mathrm{P}<0.001$ versus diabetic rats not treated with idebenone. NT, neural tube. 
a)
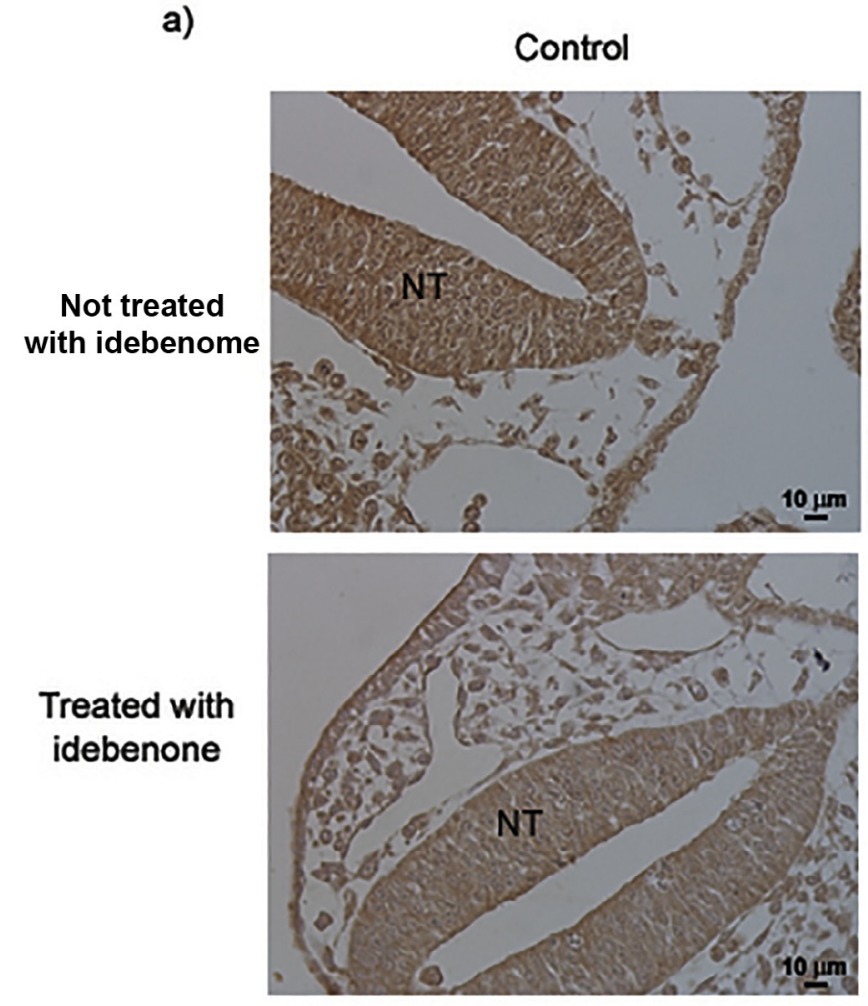

Diabetic
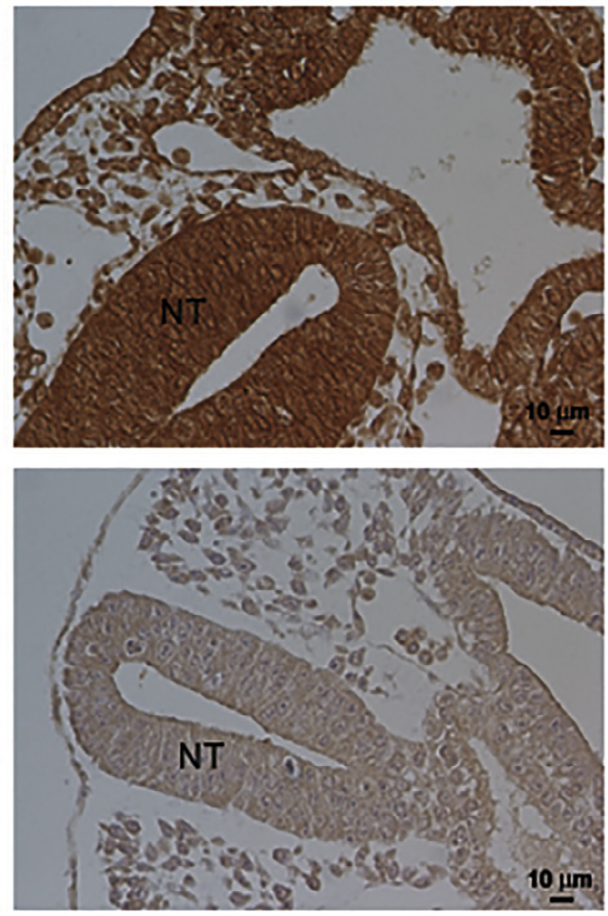

b)

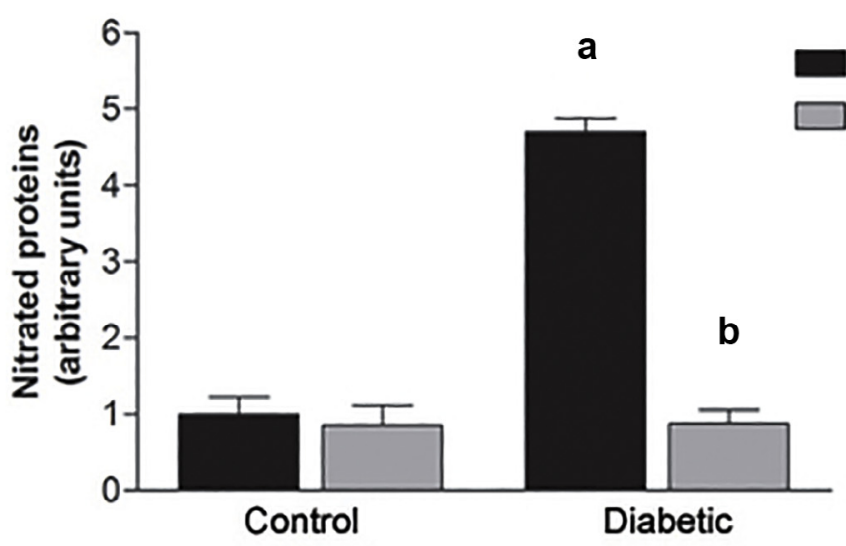

Figure 2 - Effect of maternal idebenone treatment on the levels of nitrated proteins in embryos from control and diabetic rats on day 10.5 of gestation. (a) A representative photomicrograph of a transverse section from the cephalic zone of the embryo (400X) stained with a specific anti-nitrotyrosine antibody; (b) graphs quantifying the immunohistochemical staining represented in (a). Values are means \pm SEM ( $n=5$ rats). Two-way analysis of variance in conjunction with Bonferroni's test was performed. ${ }^{\text {a }}<0.001$ versus control rats not treated with idebenone; ${ }^{b} \mathrm{P}<0.001$ versus diabetic rats not treated with idebenone NT, neural tube.

and that they were decreased in embryos from diabetic rats treated with idebenone compared with embryos from non-treated diabetic rats $(P<0.001)$ (Figure 1). We next evaluated nitrotyrosine immunostaining, a marker of the damage induced by peroxynitrites, and found that the levels of nitrated proteins in embryos from mild diabetic rats were increased compared with embryos from control rats $(P<0.001)$ (Figure 2), and they were decreased in embryos from diabetic rats treated with idebenone compared with embryos from non-treated diabetic rats $(P<0.001)$ (Figure 2$)$. As peroxynitrites in- crease the levels and activity of matrix metalloproteinases (MMP) (Capobianco et al., 2012), we were next interested in studying MMP9 levels, a marker of an inflammatory state when they are in excess. MMP9 immunostaining was increased in embryos from mild diabetic rats compared with embryos from control rats $(P<0.001)$ (Figure 3), and were decreased in embryos from both diabetic $(P<0.001)$ and control $(P<0.05)$ rats treated with idebenone compared with embryos from non-treated diabetic and control rats (Figure 3). 
a)

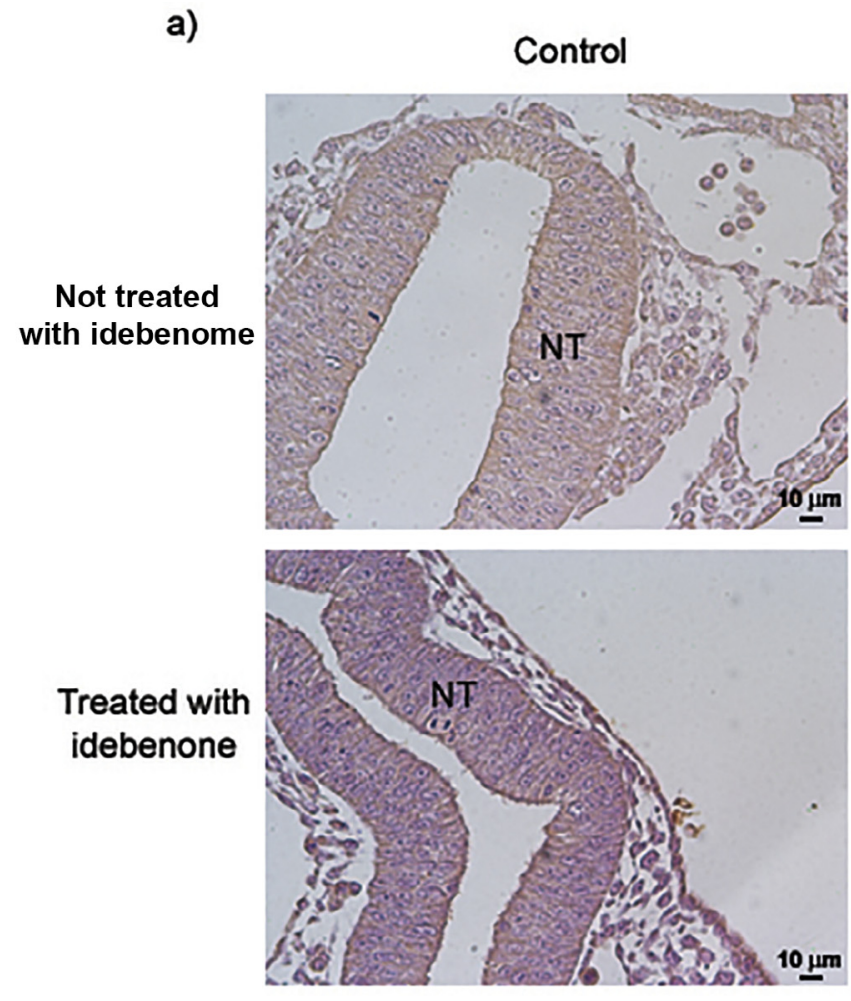

Diabetic
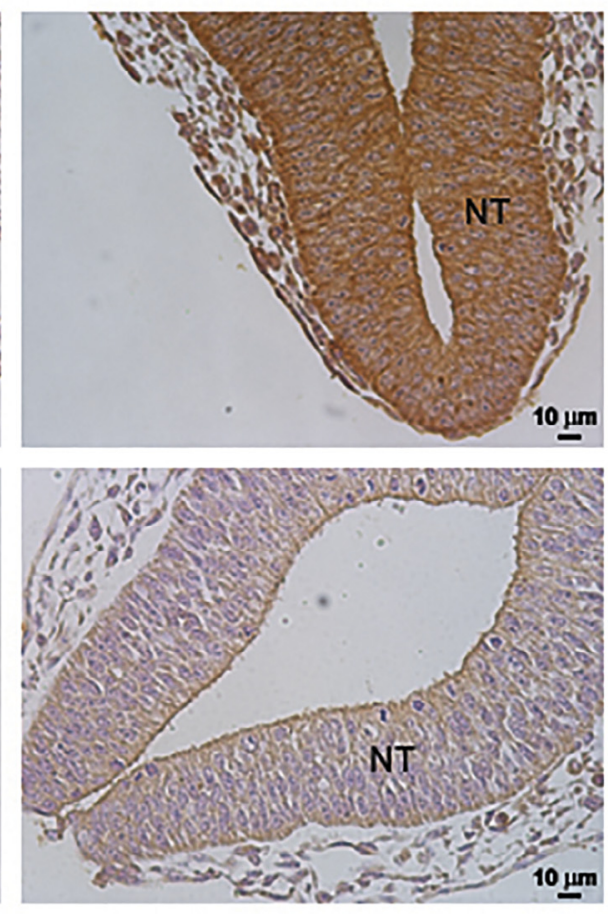

b)

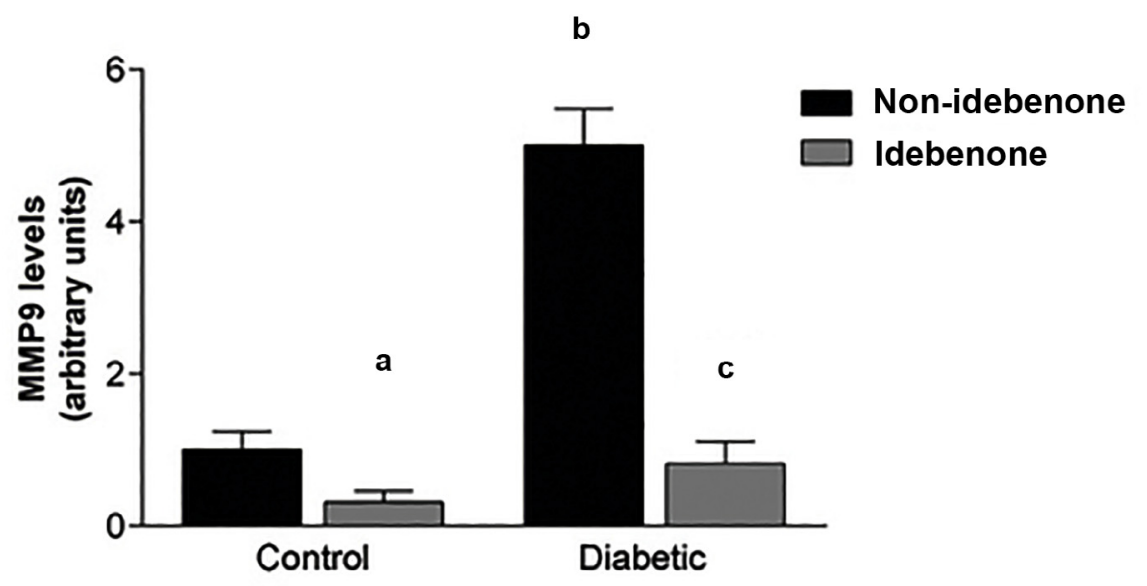

Figure 3 - Effect of maternal idebenone treatment on MMP9 levels in embryos from control and diabetic rats on day 10.5 of gestation. (a) A representative photomicrograph of a transverse section from the cephalic zone of the embryo (400X) stained with a specific anti-MMP9 antibody; (b) graphs quantifying the immunohistochemical staining represented in (a). Values are means \pm SEM ( $n=5$ rats). Two-way analysis of variance in conjunction with Bonferroni's test was performed. ${ }^{a} P<0.05$; ${ }^{b} P<0.001$ versus control rats not treated with idebenone; ${ }^{c} P<0.001$ versus diabetic rats not treated with idebenone. NT, neural tube.

Markers of mitochondrial function and biogenesis in embryos from control and diabetic rats treated or not with idebenone

With COX, the last component of the mitochondrial respiratory chain, we found reduced levels in embryos from diabetic rats compared with embryos from control rats $(P<0.05)$ (Figure 4), and increased levels in embryos from both diabetic $(P<0.001)$ and control $(P<0.05)$ rats treated with idebenone (Figure 4$)$ compared with embryos from non-treated diabetic and control rats. The subunit IVi1 of COX (COX IVi1) is involved in the regulation of COX activity. mRNA levels of Cox IVi1 in embryos from diabetic rats were decreased compared with embryos from control rats $(P<0.05)$ (Figure 5a), and increased in embryos from both diabetic $(P<0.05)$ and control $(P<0.001)$ rats treated with idebenone when respectively compared with embryos from non-treated diabetic and control rats (Figure 5a). 
a)

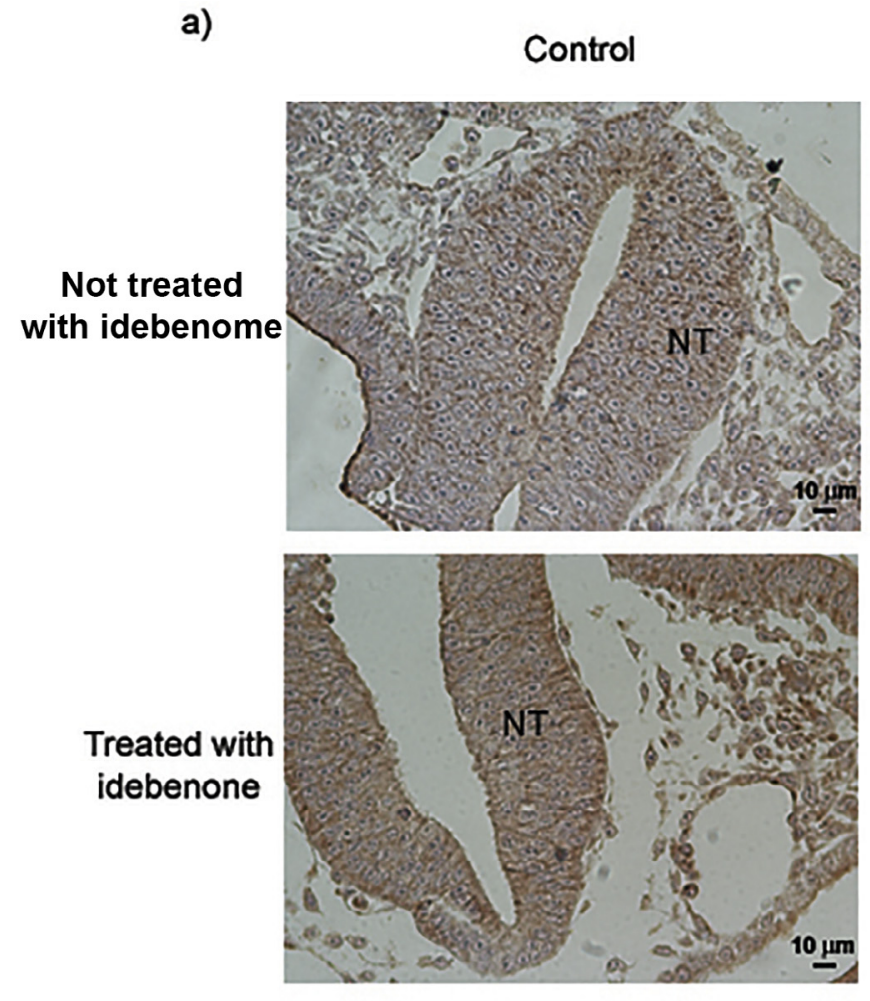

Diabetic
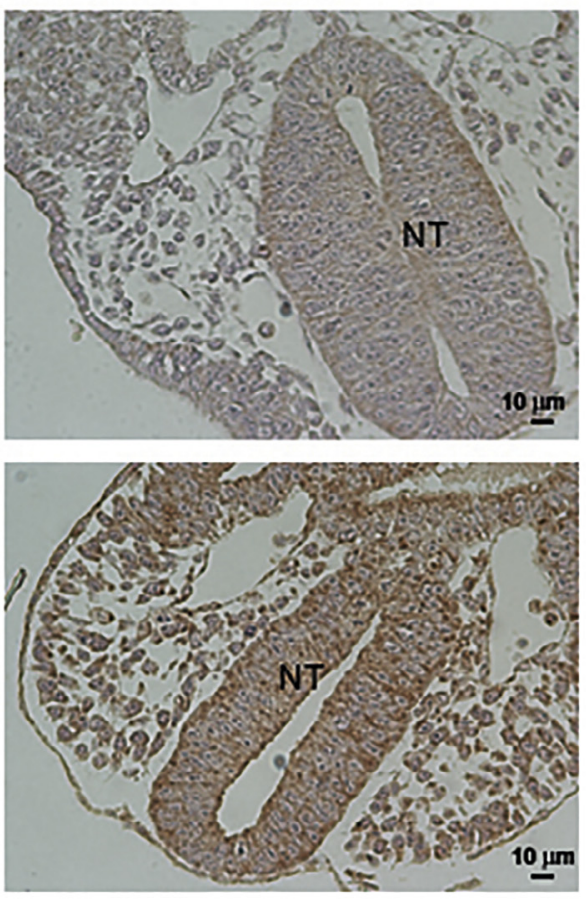

b)

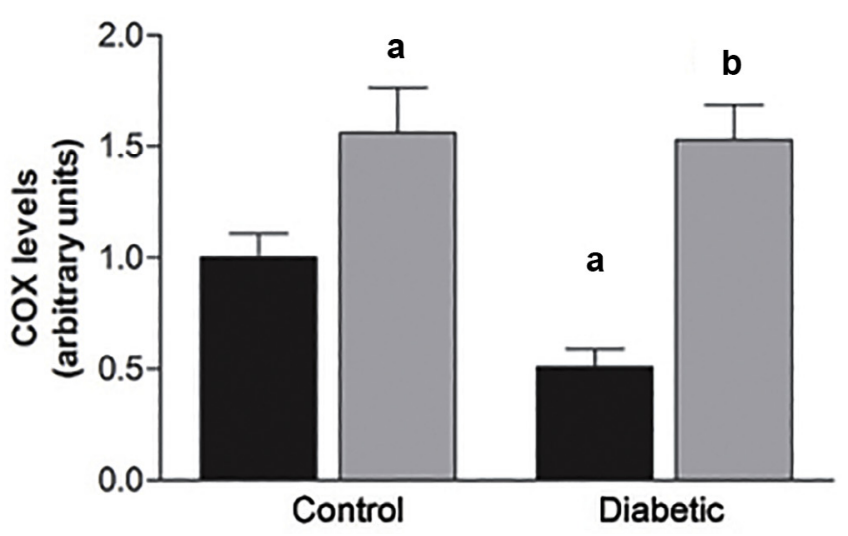

Non-idebenone Idebenone

Figure 4 - Effect of maternal idebenone treatment on cytochrome c oxidase (COX) IV levels in embryos from control and diabetic rats on day 10.5 of gestation. (a) A representative photomicrograph of a transverse section from the cephalic zone of the embryo (400X) stained with a specific anti-COX IV antibody; (b) graphs quantifying the immunohistochemical staining represented in (a). Values are means \pm SEM ( $n=5$ rats). Two-way analysis of variance in conjunction with Bonferroni's test was performed. ${ }^{a}<0.05$ versus control rats not treated with idebenone; ${ }^{b} P<0.001$ versus diabetic rats not treated with idebenone. NT, neural tube.

We also studied TFAM and NRF1, regulatory proteins involved in respiratory chain function. Tfam mRNA levels were not different in embryos from diabetic rats treated or not with idebenone when respectively compared with control rats (Figure 5b). On the other hand, Nrf1 mRNA levels were reduced in embryos from mild diabetic rats compared with embryos from control rats $(P<0.05)$ (Figure $5 c)$, and increased in embryos from diabetic rats treated with idebenone compared with embryos from non-treated diabetic rats $(P<0.05)$ (Figure 5c).

As NRF1 transcription is regulated by PGC- $1 \alpha$, we also studied this coactivator. Pgc-1 $\alpha$ mRNA levels were reduced in embryos from diabetic rats compared with embryos from control rats $(P<0.05)$ (Figure 6a) and increased in embryos from diabetic rats treated with idebenone compared with embryos from non-treated diabetic rats ( $P$ $<0.05$ ) (Figure 6a).

The nuclear receptor PPARS is involved in antioxidant and developmental processes, and is coactivated by PGC- $1 \alpha$. mRNA levels of Ppar $\delta$ were also reduced in embryos from mild diabetic rats compared with embryos from control rats $(P<0.05)$ (Figure $6 b)$ and increased in embryos from diabetic rats treated with idebenone compared with embryos from non-treated diabetic rats $(P<0.05)$ (Figure 6b). 
a)

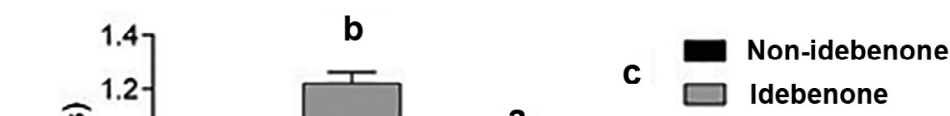

b)

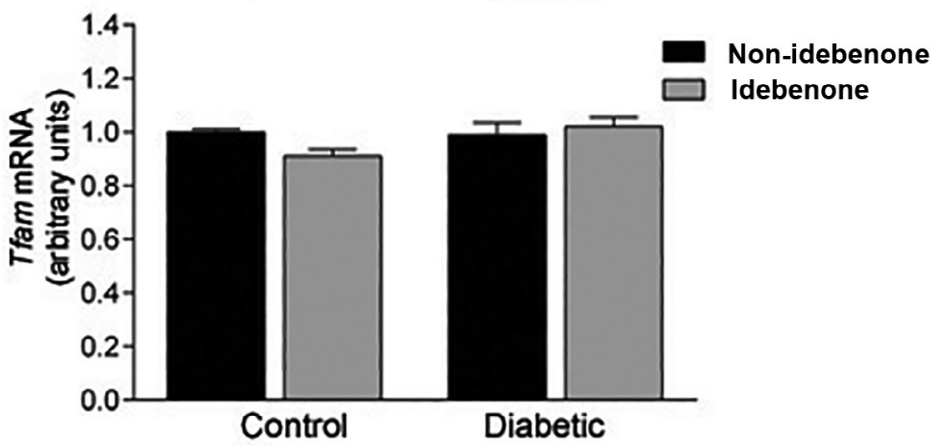

c)

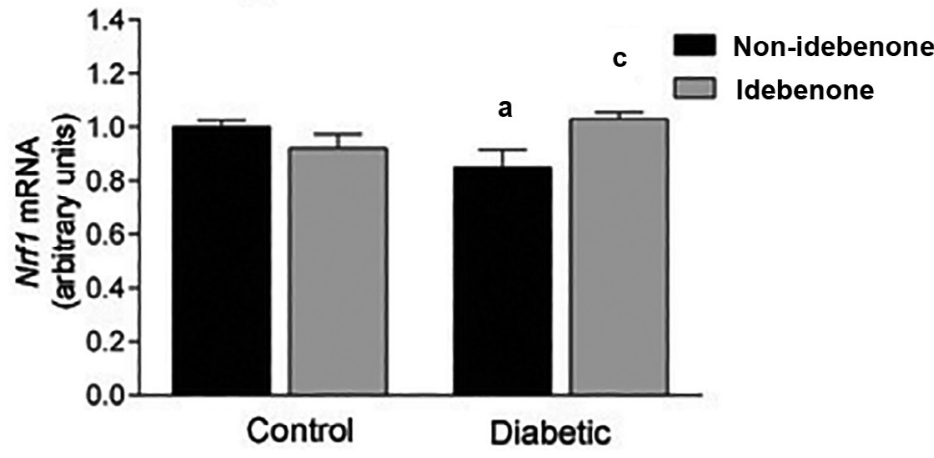

Figure 5 - Effect of maternal idebenone treatment on markers of mitochondrial function and biogenesis in embryos from control and diabetic rats on day 10.5 of gestation. (a) Cox IVi1 mRNA levels relative to those of $L 30$ in embryos from control and diabetic rats; (b) Tfam mRNA levels relative to those of $L 30$ in embryos from control and diabetic rats; (c) Nrf1 mRNA levels relative to those of $L 30$ in embryos from control and diabetic rats. Values are means \pm SEM ( $n=9$ rats). Two-way analysis of variance in conjunction with Bonferroni's test was carried out. ${ }^{\mathrm{a} P}<0.05 ;{ }^{\mathrm{b}} \mathrm{P}<0.001$ versus control rats not treated with idebenone; ${ }^{\mathrm{c}} \mathrm{P}<0.05$ versus diabetic rats not treated with idebenone.

\section{Apoptosis in embryos from control and diabetic rats treated with idebenone}

To study the effect of idebenone on apoptosis, we conducted a TUNEL assay and found that the number of apoptotic cells increased in embryos from diabetic rats compared with embryos from control rats $(P<0.001)$ (Figure 7) and reduced in embryos from diabetic rats treated with idebenone compared with embryos from non-treated diabetic rats $(P<0.05)$ (Figure 7).

\section{Discussion}

The main finding of this work was that the antioxidant idebenone can prevent the increases in markers of prooxidant and proinflammatory processes in embryos from mild diabetic rats during organogenesis. The maternal treatment with idebenone was also able to prevent the alterations in markers of mitochondrial biogenesis and function, and cell apoptosis observed in embryos of mild diabetic rats highlighting the relevance of aberrant mitochondrial pathways in diabetic embryopathy and pointing to idebenone as a putative treatment to ameliorate the development of viable embryos in a prooxidant/ proinflammatory environment.

Importantly, the increased levels of prooxidant and proinflammatory markers we observed in the embryo from mild diabetic rats during early organogenesis have also been observed in fetuses (Pustovrh et al., 2005), placentae (Martinez et al., 2012) and in the postnatal heart (Capobianco et al., 2015), suggesting that embryonic alterations may affect later development.

Moreover, we recently found that maternal idebenone administration during diabetic pregnancies prevents the programming of diabetes-induced cardiac alterations in the offspring (Higa et al., 2017).

Idebenone is a synthetic antioxidant that can be orally administered. It is found both in the cellular cytosol and inside mitochondria, and can act as a free-radical scavenger, undergoing reversible reduction - oxidation reactions to mediate mitochondrial electron flow 
a)

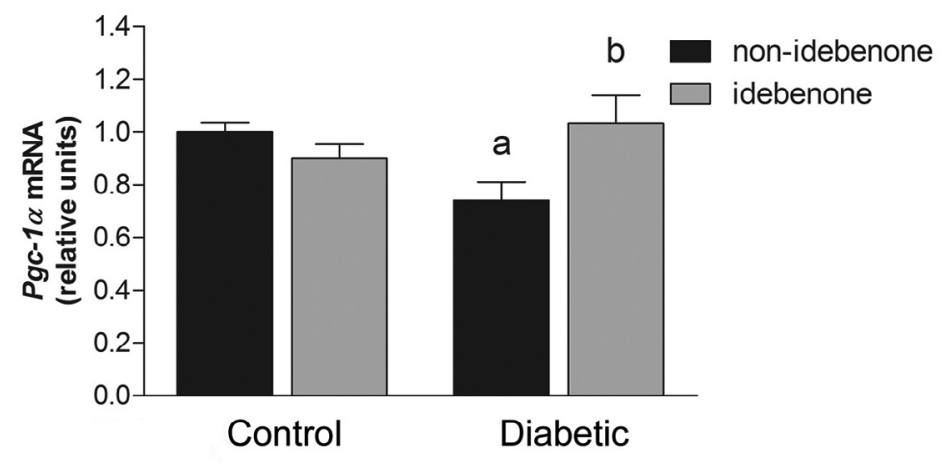

b)

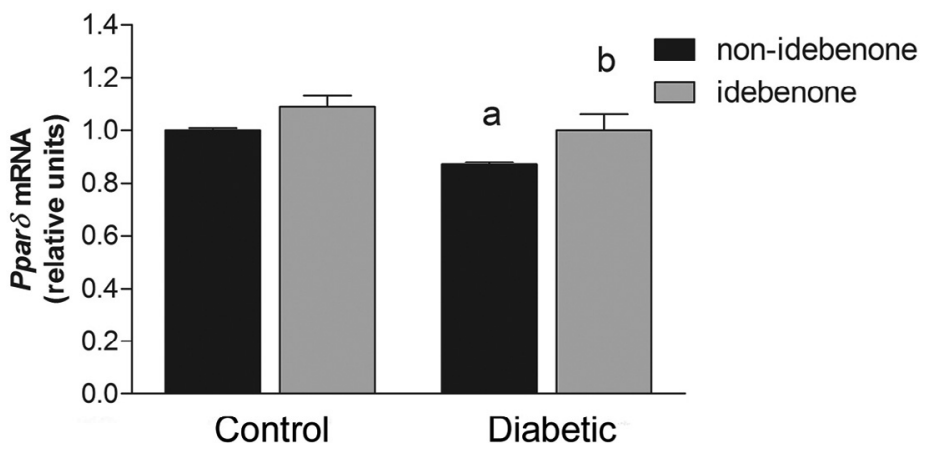

Figure 6 - Effect of maternal idebenone treatment on key regulators of cell energy homeostasis in embryos from control and diabetic rats on day 10.5 of gestation. (a) Pgc- $1 \alpha$ mRNA levels relative to those of $L 30$ in embryos from control and diabetic rats; (b) Ppar $\delta$ mRNA levels relative to those of $L 30$ in embryos from control and diabetic rats. Values are means $\pm S E M(n=9$ rats). Two-way analysis of variance in conjunction with Bonferroni's test was performed. ${ }^{\text {a }} \mathrm{P}<0.05$ versus control rats not treated with idebenone; ${ }^{\text {b }} \mathrm{P}<0.05$ versus diabetic rats not treated with idebenone.

and thus increase the production of adenosine triphosphate (Carbone et al., 2012). In this work, idebenone treatment during early organogenesis reduced the increased lipid peroxidation associated with mild diabetes, suggesting that it reduces oxidative stress, and also the increased nitrated proteins, suggesting that it reduces peroxynitriteinduced damage in embryos from diabetic rats. We have previously found increased peroxynitrites-induced damage in embryos from severe diabetic rats (glucose concentrations over $350 \mathrm{mg} / \mathrm{dl}$ ) (Jawerbaum et al., 2005), but this is the first time that this damage is reported in embryos from rats with mild hyperglycaemia Iglucose concentrations below $250 \mathrm{mg} / \mathrm{dl}$ ). Peroxynitrites are produced by the reaction between nitric oxide and superoxide and are strong oxidizing and nitrating species that promote oxidative damage by many mechanisms (Szabo, 2003). These compounds can increase the production and activation of MMP (Capobianco et al., 2012; Sugiura et al., 2012). We have previously found increased MMP9 levels and activation in embryos from severe diabetic rats (Higa et al., 2011). In this work, we also found increased MMP9 levels in the embryos from mild diabetic rats, which were reduced by idebenone. In pathological conditions, the excess of peroxynitrites can alter the mitochondrial chain function via the oxidative inactivation of complexes I, II and V and can also nitrate cytochrome c (Cassina and Radi, 1996; Cassina et al., 2000). In pathological conditions, peroxynitrites can also increase the $\mathrm{Km}$ for oxygen of COX (Cooper et al., 2003). Maternal diabetes in rats has been shown to induce alterations in mitochondrial morphology in embryos from 9 to 11 days of gestation (Yang et al., 1995). In the present study, by evaluating embryos on day 10.5 of gestation, we found decreased expression of COX in embryos from diabetic rats, and that idebenone treatment prevented this alteration, suggesting that, despite their dependence on glycolytic pathways at the developmental stage evaluated here, embryos may benefit from idebenone treatment. An increase in COX expression mediated by idebenone has been previously seen in the hippocampus and cerebellum of a mouse model of Angelman syndrome with mitochondrial dysfunction (Llewellyn et al., 2015). Interestingly, the mechanisms of COX upregulation seem to depend on upstream regulators, given that the mRNA levels of both Nrf1 (which regulates COX IV transcription) and its coactivator, Pgc-1 $\alpha$ (Puigserver and Spiegelman, 2003; Scarpulla, 2008), are upregulated with the maternal idebenone treatment in the embryos from diabetic rats. The relevance of maternal idebenone treatment as an upregulator of $\mathrm{Pgc}-1 \alpha$ expression in the embryos is supported by recent findings showing that maternal diabetes induces downregulation of $\mathrm{Pgc}-1 \alpha$ expression in the embryos, changes related to an increased rate of neural tube defects (Chen et al., 2017).

The nuclear receptor PPAR $\delta$ participates in cell proliferation and differentiation in adult tissue as well as in fetal tissues and has antiinflammatory properties (Coll et al., 2010; Rotman et al., 2006). We have previously found that aberrant PPAR $\delta$ signalling is involved in embryo dysmorphogenesis and that PPAR $\delta$ is reduced in embryos from severe diabetic rats (Higa et al., 2007, 2014). In the present study, we also found reduced Ppar $\delta$ mRNA levels in the embryos from mild diabetic rats and that idebenone was able to modulate Ppar $\delta$ transcription, increasing the reduced mRNA levels of this nuclear receptor. Although the mechanism remains unclear, it has been recently suggested that PPARs are epigenetically regulated (Lendvai et al., 2016) and that reactive oxygen species participate in many important epigenetic processes (Afanas'ev, 
a)

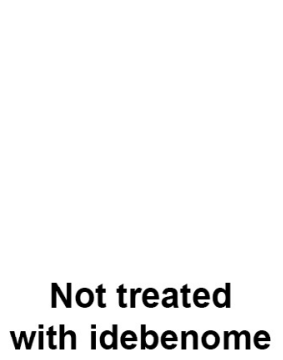

Treated with idebenone

b)
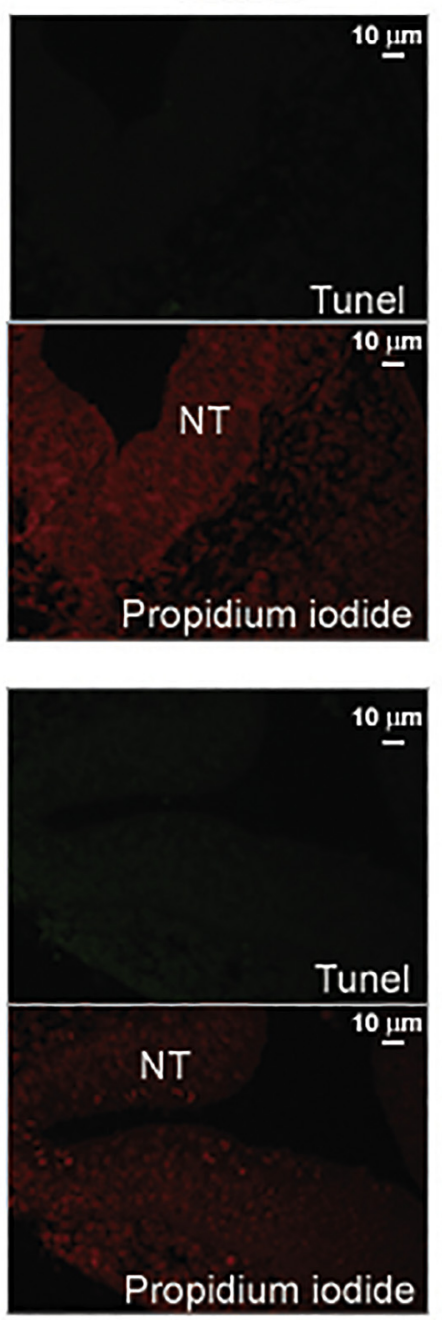

a

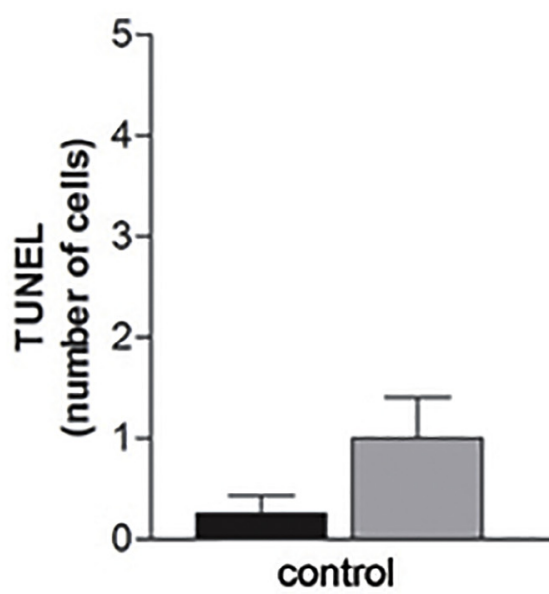

Diabetic
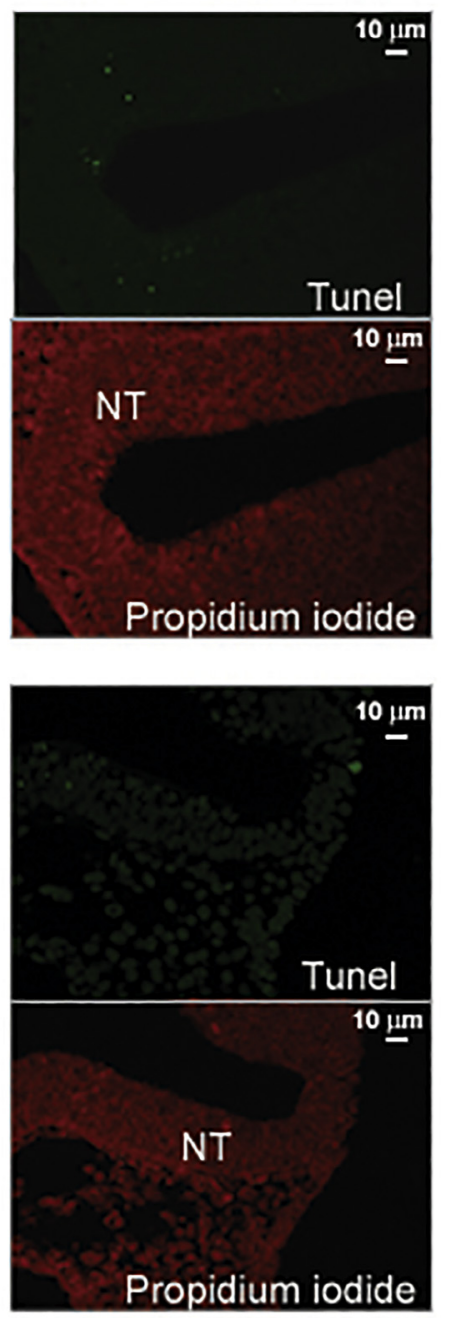

Non-idebenone

\section{Idebenone}

Figure 7 - Effect of maternal idebenone treatment on apoptosis in embryos from diabetic rats on day 10.5 of gestation. al shows representative photomicrographs of a transverse section from the cephalic zone of the embryo (400X). Apoptotic cells were labelled in green and nuclei in red by propidium iodide; b) the number of TdT (terminal deoxynucleotidyl transferase)-mediated dUTP nick-endlabelling-positive cells is shown. Values are means \pm SEM ( $n=5$ rats). Two-way analysis of variance in conjunction with Bonferroni's test was performed. ${ }^{\mathrm{P}}<0.001$ versus control rats not treated with idebenone; ${ }^{\mathrm{b}} \mathrm{P}<0.05$ versus diabetic rats not treated with idebenone. 
2015). The upregulation of Ppar $\delta$ by idebenone observed in the embryos from diabetic rats may contribute to a decrease in the prooxidant/ proinflammatory environment as PPARs regulate the expression of antioxidant enzymes and modulate the expression of the inducible isoform of nitric oxide synthase and MMPs (Barlaka et al., 2015; Chao et al., 2014). PPAR $\delta$ is also involved in inhibiting apoptosis in different tissues (Barlaka et al., 2015; Schuster et al., 2010).

An optimal balance between cell proliferation and apoptosis is required for proper development. An increased apoptotic rate related to a prooxidant-proinflammatory environment has been reported in murine embryos exposed to a diabetic environment (Gareskog et al., 2007; Wu et al., 2016; Yang et al., 2008). In the present study, idebenone administration reduced the increased apoptotic rate in embryos from diabetic rats, probably by increasing PPAR $\delta$ levels, reducing the prooxidant-proinflammatory processes, and preventing the alteration in mitochondrial function and biogenesis parameters. As a limitation, we were not able to measure idebenone levels in the embryo. Therefore, it is not clear whether the effects of maternal idebenone treatment on embryos is attributable to a direct effect on the embryos or to its impact on maternal tissues.

We conclude that oxidative stress is a main inductor of aberrant pathways in the embryo during early organogenesis and that the use of an antioxidant that targets mitochondria ameliorates several mitochondrial, nuclear, cytoplasmic and extracellular parameters, thus precluding embryonic changes associated with maternal diabetes. Idebenone is currently used in Friedreich's ataxia, Alzheimer's disease and Leber hereditary optic neuropathy disease (Gutzmann and Hadler, 1998; Meyerson et al., 2015; Parkinson et al., 2013). Therefore, it would be interesting to study whether maternal idebenone treatment may improve diabetes-induced neural alterations in the offspring. As our data suggest a potential role of idebenone in preventing maternal diabetes-induced embryo damage, clinical studies are needed to address the potential use of this drug in pregestational diabetic patients.

\section{Acknowledgement}

This work was supported by Grants from Agencia de Promoción Científica y Tecnológica of Argentina (PICT 2014-411 and PICT 20150666) and GlaxoSmithKine (PICTO-GSK 2012-0054). We thank Vet. Marcela Márquez and Tech. Enzo Cuba for their important assistance with the animal handling and Dr Dante Paz for his valuable help with the TUNEL assay.

\section{A R T I C L E I N F O}

\section{Article history:}

Received 13 September 2017

Received in revised form 10 May 2018

Accepted 10 May 2018

Declaration: The authors report no

financial or commercial conflicts of

interest.

\section{Keywords:}

Diabetes

Early organogenesis

Embryo

Idebenone

\section{REFEREN CES}

Afanas'ev, I., 2015. Mechanisms of superoxide signaling in epigenetic processes: relation to aging and cancer. Aging. Dis. 6, 216-227.

Akazawa, S., 2005. Diabetic embryopathy: studies using a rat embryo culture system and an animal model. Congenit. Anom. (Kyoto) 45, 73-79.

Barlaka, E., Gorbe, A., Gaspar, R., Paloczi, J., Ferdinandy, P., Lazou, A., 2015. Activation of PPARbeta/delta protects cardiac myocytes from oxidative stress-induced apoptosis by suppressing generation of reactive oxygen/nitrogen species and expression of matrix metalloproteinases. Pharmacol. Res. 95-96, 102-110.

Blake, R., Trounce, I.A., 2014. Mitochondrial dysfunction and complications associated with diabetes. Biochim. Biophys. Acta 1840, 1404-1412.

Braissant, O., Wahli, W., 1998. Differential expression of peroxisome proliferator-activated receptor-alpha, -beta, and-gamma during rat embryonic development. Endocrinology 139, 2748-2754.

Capobianco, E., White, V., Sosa, M., Di Marco, I., Basualdo, M.N., Faingold, M.C., Jawerbaum, A., 2012. Regulation of matrix metalloproteinases 2 and 9 activities by peroxynitrites in term placentas from type 2 diabetic patients. Reprod. Sci. 19, 814-822.

Capobianco, E., Pelesson, M., Careaga, V., Fornes, D., Canosa, I., Higa, R., Maier, M., Jawerbaum, A., 2015. Intrauterine programming of lipid metabolic alterations in the heart of the offspring of diabetic rats is prevented by maternal diets enriched in olive oil. Mol. Nutr. Food Res. 59, 1997-2007.

Carbone, C., Pignatello, R., Musumeci, T., Puglisi, G., 2012. Chemical and technological delivery systems for idebenone: a review of literature production. Expert Opin. Drug Deliv. 9, 1377-1392.

Cassina, A., Radi, R., 1996. Differential inhibitory action of nitric oxide and peroxynitrite on mitochondrial electron transport. Arch. Biochem. Biophys. 328, 309-316.

Cassina, A.M., Hodara, R., Souza, J.M., Thomson, L., Castro, L., Ischiropoulos, H., Freeman, B.A., Radi, R., 2000. Cytochrome c nitration by peroxynitrite. J. Biol. Chem. 275, 21409-21415.

Chao, X., Xiong, C., Dong, W., Qu, Y., Ning, W., Liu, W., Han, F., Ma, Y., Wang, R., Fei, Z., Han, H., 2014. Activation of peroxisome proliferator-activated receptor beta/delta attenuates acute ischemic stroke on middle cerebral ischemia occlusion in rats. J. Stroke Cerebrovasc. Dis. 23, 1396-1402.

Chen, X., Zhong, J., Dong, D., Liu, G., Yang, P., 2017. Endoplasmic Reticulum Stress-Induced CHOP Inhibits PGC-1alpha and Causes Mitochondrial Dysfunction in Diabetic Embryopathy. Toxicol. Sci. 158, 275-285.

Coll, T., Barroso, E., Alvarez-Guardia, D., Serrano, L., Salvado, L., Merlos, M., Palomer, X., Vazquez-Carrera, M., 2010. The role of peroxisome proliferator-activated receptor beta/delta on the inflammatory basis of metabolic disease. PPAR Res. 2010, 368467.

Cooper, C.E., Davies, N.A., Psychoulis, M., Canevari, L., Bates, T.E., Dobbie, M.S., Casley, C.S., Sharpe, M.A., 2003. Nitric oxide and peroxynitrite cause irreversible increases in the $K(\mathrm{~m})$ for oxygen of mitochondrial cytochrome oxidase: in vitro and in vivo studies. Biochim. Biophys. Acta 1607, 27-34.

Dhar, S.S., Ongwijitwat, S., Wong-Riley, M.T., 2008. Nuclear respiratory factor 1 regulates all ten nuclear-encoded subunits of cytochrome $\mathrm{c}$ oxidase in neurons. J. Biol. Chem. 283, 3120-3129.

Eleazu, C.O., Eleazu, K.C., Chukwuma, S., Essien, U.N., 2013. Review of the mechanism of cell death resulting from streptozotocin challenge in experimental animals, its practical use and potential risk to humans. J. Diabetes. Metab. Disord. 12, 60.

Eriksson, U.J., Wentzel, P., 2016. The status of diabetic embryopathy. Ups. J. Med. Sci. 121, 96-112.

Gareskog, M., Eriksson, U.J., Wentzel, P., 2006. Combined supplementation of folic acid and vitamin E diminishes diabetesinduced embryotoxicity in rats. Birth Defects Res. A. Clin Mol. Teratol. 76, 483-490. 
Gareskog, M., Cederberg, J., Eriksson, U.J., Wentzel, P., 2007. Maternal diabetes in vivo and high glucose concentration in vitro increases apoptosis in rat embryos. Reprod. Toxicol. 23, 63-74.

Geromel, V., Darin, N., Chretien, D., Benit, P., DeLonlay, P., Rotig, A., Munnich, A., Rustin, P., 2002. Coenzyme Q(10) and idebenone in the therapy of respiratory chain diseases: rationale and comparative benefits. Mol. Genet. Metab. 77, 21-30.

Gutzmann, H., Hadler, D., 1998. Sustained efficacy and safety of idebenone in the treatment of Alzheimer's disease: update on a 2-year double-blind multicentre study. J. Neural Transm. Suppl. 54, 301-310.

Higa, R., Jawerbaum, A., 2013. Intrauterine effects of impaired lipid homeostasis in pregnancy diseases. Curr. Med. Chem. 20, 23382350.

Higa, R., Gonzalez, E., Pustovrh, M.C., White, V., Capobianco, E., Martinez, N., Jawerbaum, A., 2007. PPARdelta and its activator PGI2 are reduced in diabetic embryopathy: involvement of PPARdelta activation in lipid metabolic and signalling pathways in rat embryo early organogenesis. Mol. Hum. Reprod. 13, 103-110.

Higa, R., Kurtz, M., Capobianco, E., Martinez, N., White, V., Jawerbaum, A., 2011. Altered matrix metalloproteinases and tissue inhibitors of metalloproteinases in embryos from diabetic rats during early organogenesis. Reprod. Toxicol. 32, 449-462.

Higa, R., Kurtz, M., Mazzucco, M.B., Musikant, D., White, V., Jawerbaum, A., 2012. Folic acid and safflower oil supplementation interacts and protects embryos from maternal diabetes-induced damage. Mol. Hum. Reprod. 18, 253-264.

Higa, R., Roberti, S.L., Musikant, D., Mazzucco, M.B., White, V., Jawerbaum, A., 2014. Effects of maternal dietary olive oil on pathways involved in diabetic embryopathy. Reprod. Toxicol. 49C, 185-195.

Higa, R., Roberti, S.L., Capobianco, E., Fornes, D., White, V., Jawerbaum, A., 2017. Pro-oxidant/pro-inflammatory alterations in the offspring $s$ heart of mild diabetic rats are regulated by maternal treatments with a mitochondrial antioxidant. Reprod. Toxicol. 73, 269-279.

Jawerbaum, A., Gonzalez, E., 2006. Diabetic pregnancies: the challenge of developing in a pro-inflammatory environment. Curr. Med. Chem. $13,2127-2138$

Jawerbaum, A., White, V., 2010. Animal models in diabetes and pregnancy. Endocr. Rev. 31, 680-701.

Jawerbaum, A., White, V., 2017. Review on intrauterine programming: consequences in rodent models of mild diabetes and mild fat overfeeding are not mild. Placenta 52, 21-32.

Jawerbaum, A., Higa, R., White, V., Capobianco, E., Pustovrh, C., Sinner, D., Martinez, N., Gonzalez, E., 2005. Peroxynitrites and impaired modulation of nitric oxide concentrations in embryos from diabetic rats during early organogenesis. Reproduction 130, 695-703.

Lendvai, A., Deutsch, M.J., Plosch, T., Ensenauer, R., 2016. The peroxisome proliferator-activated receptors under epigenetic control in placental metabolism and fetal development. Am. J. Physiol. Endocrinol. Metab. 310, E797-E810.

Lin, P., Liu, J., Ren, M., Ji, K., Li, L., Zhang, B., Gong, Y., Yan, C., 2015. Idebenone protects against oxidized low density lipoprotein induced mitochondrial dysfunction in vascular endothelial cells via GSK3beta/beta-catenin signalling pathways. Biochem. Biophys. Res. Commun. 465, 548-555.

Llewellyn, K.J., Nalbandian, A., Gomez, A., Wei, D., Walker, N., Kimonis, V.E., 2015. Administration of CoQ10 analogue ameliorates dysfunction of the mitochondrial respiratory chain in a mouse model of Angelman syndrome. Neurobiol. Dis. 76, 77-86.

Martinez, N., Sosa, M., Higa, R., Fornes, D., Capobianco, E., Jawerbaum, A., 2012. Dietary treatments enriched in olive and safflower oils regulate seric and placental matrix metalloproteinases in maternal diabetes. Placenta 33, 8-16.

Meyerson, C., Van Stavern, G., McClelland, C., 2015. Leber hereditary optic neuropathy: current perspectives. Clin. Ophthalmol. 9, 11651176 .
Ornoy, A., 2007. Embryonic oxidative stress as a mechanism of teratogenesis with special emphasis on diabetic embryopathy. Reprod. Toxicol. 24, 31-41.

Parkinson, M.H., Schulz, J.B., Giunti, P., 2013. Co-enzyme Q10 and idebenone use in Friedreich's ataxia. J. Neurochem. 126 (Suppl. 1), 125-141.

Puigserver, P., Spiegelman, B.M., 2003. Peroxisome proliferatoractivated receptor-gamma coactivator 1 alpha (PGC-1 alpha): transcriptional coactivator and metabolic regulator. Endocr. Rev. 24, 78-90.

Pustovrh, M.C., Jawerbaum, A., Capobianco, E., White, V., Martinez, N., Lopez-Costa, J.J., Gonzalez, E., 2005. Oxidative stress promotes the increase of matrix metalloproteinases- 2 and -9 activities in the fetoplacental unit of diabetic rats. Free Radic. Res. 39, 1285-1293.

Rotman, N., Michalik, L., Desvergne, B., Wahli, W., 2006. PPARs in fetal and early postnatal development. Adv. Dev. Biol. 16, 33-64.

Sakamaki, H., Akazawa, S., Ishibashi, M., Izumino, K., Takino, H., Yamasaki, H., Yamaguchi, Y., Goto, S., Urata, Y., Kondo, T., Nagataki, S., 1999. Significance of glutathione-dependent antioxidant system in diabetes-induced embryonic malformations. Diabetes 48, 1138 1144.

Scarpulla, R.C., 2008. Nuclear control of respiratory chain expression by nuclear respiratory factors and PGC-1-related coactivator. Ann. N. Y. Acad. Sci. 1147, 321-334

Schuster, M., Zouboulis, C.C., Ochsendorf, F., Muller, J., Thaci, D., Bernd, A., Kaufmann, R., Kippenberger, S., 2010. Peroxisome proliferator-activated receptor activators protect sebocytes from apoptosis: a new treatment modality for acne? Br. J. Dermatol. 164, 182-186.

Singh, C.K., Kumar, A., Hitchcock, D.B., Fan, D., Goodwin, R., LaVoie, H.A., Nagarkatti, P., DiPette, D.J., Singh, U.S., 2011. Resveratrol prevents embryonic oxidative stress and apoptosis associated with diabetic embryopathy and improves glucose and lipid profile of diabetic dam. Mol. Nutr. Food Res. 55, 1186-1196.

Sugiura, H., Kawabata, H., Ichikawa, T., Koarai, A., Yanagisawa, S., Kikuchi, T., Minakata, Y., Matsunaga, K., Nakanishi, M., Hirano, T., Akamatsu, K., Furukawa, K., Ichinose, M., 2012. Inhibitory effects of theophylline on the peroxynitrite-augmented release of matrix metalloproteinases by lung fibroblasts. Am. J. Physiol. Lung Cell. Mol. Physiol. 302, L764-L774.

Szabo, C., 2003. Multiple pathways of peroxynitrite cytotoxicity. Toxicol. Lett. 140-141, 105-112.

Viana, M., Castro, M., Barbas, C., Herrera, E., Bonet, B., 2003. Effect of different doses of vitamin $E$ on the incidence of malformations in pregnant diabetic rats. Ann. Nutr. Metab. 47, 6-10.

Wang, Y.X., Lee, C.H., Tiep, S., Yu, R.T., Ham, J., Kang, H., Evans, R.M., 2003. Peroxisome-proliferator-activated receptor delta activates fat metabolism to prevent obesity. Cell 113, 159-170.

Wu, Y., Reece, E.A., Zhong, J., Dong, D., Shen, W.B., Harman, C.R., Yang, P., 2016. Type 2 diabetes mellitus induces congenital heart defects in murine embryos by increasing oxidative stress, endoplasmic reticulum stress, and apoptosis. Am. J. Obstet. Gynecol. 215, 366, e1-366.e10.

Yang, P., Zhao, Z., Reece, E.A., 2008. Activation of oxidative stress signaling that is implicated in apoptosis with a mouse model of diabetic embryopathy. Am. J. Obstet. Gynecol. 198, e131-e137.

Yang, X., Borg, L.A., Eriksson, U.J., 1995. Altered mitochondrial morphology of rat embryos in diabetic pregnancy. Anat. Rec. 241, 255-267.

Zabihi, S., Loeken, M.R., 2010. Understanding diabetic teratogenesis: where are we now and where are we going? Birth Defects Res. A. Clin Mol. Teratol. 88, 779-790.

Zaken, V., Kohen, R., Ornoy, A., 2000. The development of antioxidant defense mechanism in young rat embryos in vivo and in vitro. Early Pregnancy 4, 110-123.

Zhao, Z., Reece, E.A., 2013. New concepts in diabetic embryopathy. Clin Lab. Med. 33, 207-233. 